\title{
THE STRUCTURE OF SPACES OF $\mathbb{R}$-PLACES OF RATIONAL FUNCTION FIELDS OVER REAL CLOSED FIELDS
}

\author{
KATARZYNA KUHLMANN
}

\begin{abstract}
For arbitrary real closed fields $R$, we study the structure of the space $M(R(y))$ of $\mathbb{R}$-places of the rational function field in one variable over $R$ and determine its dimension to be 1 . We determine small subbases for its topology and discuss a suitable metric in the metrizable case. In the case of non-archimedean $R$, we exhibit the rich variety of homeomorphisms of subspaces that can be found in such spaces.
\end{abstract}

1. Introduction. Let $\mathcal{X}(K)$ be the space of orderings, given in the form of positive cones (not containing 0 ), of a formally real field $K$, and let $M(K)$ be the space of $\mathbb{R}$-places of $K$, i.e., places $\xi: K \rightarrow \overline{\mathbb{R}}$, where $\overline{\mathbb{R}}=\mathbb{R} \cup\{\infty\}$, i.e., a circle. It is well known that $\mathcal{X}(K)$ is a Boolean space (i.e., it is compact, Hausdorff and totally disconnected) under the Harrison topology given by the subbasis of sets

$$
H(a):=\{P \in \mathcal{X}(K) \mid a \in P\}, \quad a \in K \backslash\{0\} .
$$

The natural surjection

$$
\lambda: \mathcal{X}(K) \longrightarrow M(K)
$$

makes $M(K)$ (with the quotient topology) a compact Hausdorff space. A subbasis for the quotient topology on $M(K)$ is given by the family of open sets of the form

$$
U(a)=\left\{\xi \in M(K) \mid \xi(a) \in \mathbb{R}^{+}\right\}, \quad a \in K .
$$

Here, " $\xi(a) \in \mathbb{R}^{+}$" means that $\infty \neq \xi(a)>0$. For details, see [9]. In the present paper, the quotient topology will be called the Harrison topology on $M(K)$.

2010 AMS Mathematics subject classification. Primary 13J30, Secondary 12J15, $12 \mathrm{~J} 25,54 \mathrm{E} 45$.

Keywords and phrases. Real places, spaces of real places.

Received by the editors on April 26, 2013, and in revised form on June 16, 2014. 
Let $R$ be a real closed field. The unique ordering $\dot{R}^{2}$ of $R$ determines a natural valuation

$$
v: R \longrightarrow v R \cup\{\infty\}
$$

with $v R$ a divisible ordered abelian group. This turns $R$ into an ultrametric space, where the ultrametric distance $u$ is defined as follows:

$$
u(a, b)=v(a-b) .
$$

Take any cut $(S, T)$ in $v R$. The ultrametric ball with center at $a \in R$ and radius $T$ is defined as:

$$
B_{T}(a)=\{b \in R: v(b-a) \in T \cup\{\infty\}\} .
$$

If $B_{1}$ and $B_{2}$ are ultrametric balls in $R$, then one of the following holds: $B_{1} \subset B_{2}, B_{2} \subset B_{1}$ or $B_{1} \cap B_{2}=\emptyset$. Every ball $B$ determines two cuts in $R: B^{-}$with the lower cut set $\{a \in R \mid a<B\}$ and $B^{+}$with the upper cut set $\{a \in R \mid a>B\}$.

Throughout this paper, $R(y)$ will always denote the rational function field in one variable over the field $R$. Let $\mathcal{C}(R)$ be the set of cuts in $R$ (which includes the improper cuts $(\emptyset, R)$ and $(R, \emptyset)$ ).

Proposition 1.1. If $R$ is a real closed field, then there is a bijection $\chi$ from $\mathcal{C}(R)$ onto $\mathcal{X}(R(y))$ given by

$$
\begin{aligned}
(D, E) & \longmapsto P, \text { where } \\
P & =\left\{f \in R(y) \mid \exists_{d \in D \cup\{-\infty\}} \exists_{e \in E \cup\{\infty\}} \forall_{c \in(d, e)} f(c)>0\right\}
\end{aligned}
$$

for $(D, E) \in \mathcal{C}(R)$. The inverse mapping is

$$
P \longmapsto\left(D_{P}, E_{P}\right)
$$

where $D_{P}=\{d \in R \mid d<y\}$ and $E_{P}=\{e \in R \mid y<e\}$ for $P \in \mathcal{X}(R(y))$.

Indeed, it is well known that, since $R$ is a real closed field, the mapping (1.3) is a bijection, and it is an easy exercise to prove that the set $P$ in (1.2) is a positive cone. It contains $y-c$ if and only if $c \in D$, and therefore, $P$ is the unique positive cone that maps to $(D, E)$ via the mapping in (1.3). 
The set of cuts of an ordered set is an ordered set, so it carries the order topology. In fact, the bijection given above is a homeomorphism between the spaces $\mathcal{C}(R)$ with order topology and $\mathcal{X}(R(y))$ with Harrison topology. For details, see [8]. In that paper, we also gave a handy criterion for two orderings on $R(y)$ to be sent to the same $\mathbb{R}$-place by $\lambda$. We say that $C_{1}$ and $C_{2}$ are equivalent cuts if $C_{1}=C_{2}$ or $\left\{C_{1}, C_{2}\right\}=\left\{B^{-}, B^{+}\right\}$for some ultrametric ball $B$ in $R$.

Theorem 1.2. Take a real closed field $R$ and two distinct orderings $P_{1}$ and $P_{2}$ of $R(y)$. Let $C_{1}$ and $C_{2}$ be the corresponding cuts in $R$. Then $\lambda\left(P_{1}\right)=\lambda\left(P_{2}\right)$ if and only if $C_{1}$ and $C_{2}$ are equivalent.

For any $r \in R$, the set $\{r\}$ is an ultrametric ball since $\{r\}=B_{T}(r)$ for $T=\emptyset$. Also $R=B_{v R}(0)$ is an ultrametric ball. The cuts $r^{-}:=\{r\}^{-}$ and $r^{+}:=\{r\}^{+}$determined by a ball $\{r\}$ are called principal cuts. The cuts determined by the ball $R$, i.e., the cuts $(\emptyset, R)$ and $(R, \emptyset)$, are called improper cuts. The $\mathbb{R}$-place of $R(y)$ determined by principal cuts in $r$ will be called the $r$-principal place, and the place determined by improper cuts will be called the $\infty$-principal place. All these places will be called principal places. Note that, since the set of principal cuts is dense in the space $\mathcal{X}(R(y))$, the set of principal places is dense in $M(R(y))$.

In Section 2, we will show that a subbasis for the space $M(R(y))$ can be given by the rather small collection of all sets $U(f)$ where $f$ runs through all linear function and functions of the form $(y-a) /(y-b)$, all of them with coefficients in any dense subfield of $R$.

The space $M(R(y))$ is metrizable if and only if $R$ contains a countable dense subfield (see [8]). In Section 3, we will use the subbasis described above to obtain a metric on $M(R(y))$ in the metrizable case.

The problem of determining the topological dimensions of spaces of $\mathbb{R}$-places of function fields seems to be very complicated. Recently, some new results have appeared in [1] regarding the dimension of spaces of $\mathbb{R}$-places of rational function fields in several variables over totally archimedean fields. In Section 4, we determine the dimension of the space $M(R(y))$ for any (not necessarily archimedean) real closed field $R$. In general dimension theory, there are three basic notions of dimension of a topological space: the covering dimension, the small inductive 
dimension and the large inductive dimension. If a compact space is not metrizable, then the values of the various dimensions may differ. However, we prove:

Theorem 1.3. If $R$ is any real closed field, then the (small or large) inductive dimension and the covering dimension of $M(R(y))$ are 1 .

In Section 4, we will mention other known results about dimensions of spaces of $\mathbb{R}$-places of certain rational function fields.

It is well known that, for any archimedean real closed field $R$ the space $M(R(y))$ is homeomorphic to a circle. In fact, this is an easy consequence of Theorem 1.2. The latter can be employed to show the amazingly rich topological structure of $M(R(y))$ when $R$ is nonarchimedean real closed. Indeed, any non-trivial automorphism of the field $R(y)$ generates a non-trivial homeomorphism of $M(R(y))$ which reveals homeomorphisms between subspaces of this space. In particular cases, the space $M(R(y))$ can be self-homeomorphic, i.e., homeomorphic to proper subspaces. We will describe these types of homeomorphisms of $M(R(y))$ in Sections 5 and 6 .

Analyzing the gluing, we obtain in Section 6 the big picture of what we have named the "densely fractal pearl necklace." While a rich selfsimilarity structure is commonly seen as an indication for a fractal, it is usually not taken as the only criterion, However, $M(R(y))$ is compact; hence, in the metrizable case (see Section 3 ) it is automatically called "fractal" by some authors. Using the results from Sections 2 and 3, we will study in a subsequent paper in which way $M(R(y))$ meets other classical definitions of fractals. In the non-metrizable case, the use of the notion "fractal" is even more delicate, as there seems to be no authoritative literature on possible definitions in this case. We will try to make a contribution to this problem in another subsequent paper.

2. A small subbasis for $M(R(y))$. Take any real closed field $R$. Denote by $v$ the natural valuation on $R$ with value group $v R$ and by $\xi_{0}$ the corresponding unique $\mathbb{R}$-place of $R$. A subbasis of $M(R(y))$ is given by the sets

$$
U(f)=\left\{\xi \in M(R(y)) \mid \xi(f) \in \mathbb{R}^{+}\right\}, \quad f \in R(y) .
$$


In this chapter, we will present a much smaller subbasis. We need some preparations. We will say that $f \in R(y)$ is $\mathbb{Q}^{+}$-bounded on a subset $S \subseteq R$ if there are positive rational numbers $q_{1}$ and $q_{2}$ such that $q_{1} \leq f(c) \leq q_{2}$ for every $c \in S$.

Lemma 2.1. Take $f \in R(y), \xi \in M(R(y))$ and $P \in \lambda^{-1}(\xi)$. Let $(D, E)$ be the cut in $R$ corresponding to $P$. Then $\xi \in U(f)$ if and only if there are $d \in D \cup\{-\infty\}$ and $e \in E \cup\{\infty\}$ such that $f$ is $\mathbb{Q}^{+}$-bounded on $(d, e)$.

Proof. Assume first that $\xi \in U(f)$. Then there are positive rational numbers $q_{1}$ and $q_{2}$ such that $q_{1}<\xi(f)<q_{2}$. Therefore, $q_{1}<_{P} f<_{P} q_{2}$. By (1.2), there exist $d \in D \cup\{-\infty\}$ and $e \in E \cup\{\infty\}$ such that $q_{1}<f(c)<q_{2}$ holds in $R$ for every $c \in(d, e)$.

Now suppose that there are $d \in D \cup\{-\infty\}, e \in E \cup\{\infty\}$ and positive rational numbers $q_{1}$ and $q_{2}$ such that $q_{1} \leq f(c) \leq q_{2}$ for every $c \in(d, e)$. Then there are also positive rational numbers $q_{1}^{\prime}$ and $q_{2}^{\prime}$ such that $q_{1}^{\prime}<f(c)<q_{2}^{\prime}$ for every $c \in(d, e)$. This implies by (1.2) that $q_{1}^{\prime}<_{P} f<_{P} q_{2}^{\prime}$, whence $q_{1}^{\prime} \leq \xi(f) \leq q_{2}^{\prime}$ and $\xi \in U(f)$.

Corollary 2.2. Take $g \in R(y)$, and suppose that $g$ is $\mathbb{Q}^{+}$-bounded on $(d, e)$. If $\left(D^{*}, E^{*}\right)$ is a cut in $(d, e)$, i.e., $d \in D^{*} \cup\{-\infty\}$ and $E^{*} \cup\{\infty\}$, then the $\mathbb{R}$-place $\eta$ determined by the ordering corresponding to the cut $\left(D^{*}, E^{*}\right)$ is in $U(g)$.

Corollary 2.3. Assume that $f$ is a linear polynomial and $\xi \in M(R(y))$ is induced by the ball-cuts generated by a ball B. Then $\xi \in U(f)$ if and only if $f$ is $\mathbb{Q}^{+}$-bounded on $B$.

Proof. Denote by $(D, E)$ and $\left(D^{\prime}, E^{\prime}\right)$ with $D \subset D^{\prime}$ the two ball cuts induced by $B$. Then Lemma 2.1 shows that $\xi \in U(f)$ if and only if there are $d \in D \cup\{-\infty\}, e \in E, d^{\prime} \in D^{\prime}$, and $e^{\prime} \in E^{\prime} \cup\{\infty\}$ such that $f$ is $\mathbb{Q}^{+}$-bounded on $(d, e)$ and on $\left(d^{\prime}, e^{\prime}\right)$. This in turn holds if and only if $f$ is $\mathbb{Q}^{+}$-bounded on $(d, e) \cup\left(d^{\prime}, e^{\prime}\right)$.

Assume that the latter holds. Because $f$ is linear, we then have for every $b \in B$ that $f(b)$ lies between $f(d)$ and $f\left(e^{\prime}\right)$, so $f$ is $\mathbb{Q}^{+}$-bounded on $B$. 
Now assume that $f$ is $\mathbb{Q}^{+}$-bounded on $B$, that is, there are positive rational numbers $q_{1}, q_{2}$ such that $q_{1} \leq f(c) \leq q_{2}$ for every $c \in B$. Take positive rational numbers $q_{1}^{\prime}$ and $q_{2}^{\prime}$ such that $q_{1}^{\prime}<q_{1}$ and $q_{2}<q_{2}^{\prime}$. If $f$ is not constant, then define

$$
d=\min \left\{f^{-1}\left(q_{1}^{\prime}\right), f^{-1}\left(q_{2}^{\prime}\right)\right\}
$$

and

$$
e^{\prime}=\max \left\{f^{-1}\left(q_{1}^{\prime}\right), f^{-1}\left(q_{2}^{\prime}\right)\right\} .
$$

Then $d, e^{\prime} \notin B$, and therefore, $d \in D$ and $e^{\prime} \in E^{\prime}$. If $f$ is constant and $\mathbb{Q}^{+}$-bounded on the ball $B$, then $f$ is $\mathbb{Q}^{+}$-bounded on $R$, and hence, also on $(d, e) \cup\left(d^{\prime}, e^{\prime}\right)$.

Remark 2.4. The Baer-Krull theorem says that the number of orderings of the field $F$ determining the same $\mathbb{R}$-place $\xi$ is equal to the number of characters of the quotient group $V_{\xi} / 2 V_{\xi}$, where $V_{\xi}$ denotes the value group of the valuation $v_{\xi}$ originating from $\xi$. In [7], the authors described all possible value groups determined by orderings of the field $R(y)$. Either the group is equal to $v R$ (in the immediate and in the residue transcendental case), or it is isomorphic to $v R \oplus \mathbb{Z}$ (in the value transcendental case). Since $v R$ is divisible, $\xi$ is determined by a single ordering if $V_{\xi}=v R$, and by two orderings if $V_{\xi}=v R \oplus \mathbb{Z}$. On the other hand, Theorem 1.2 gives us that $\xi \in M(R(y))$ is determined by a single ordering if and only if the cut corresponding to this ordering is a non-ball cut. We conclude that $V_{\xi}=v R \oplus \mathbb{Z}$ if $\xi$ is induced by a ball cut, and $V_{\xi}=v R$ otherwise.

If $\xi \in M(R(y))$ is induced by the ball-cuts generated by a ball $B_{T}(a)$, then by [8, Lemma 2.6], we have that

$$
v(a-d)>v_{\xi}(y-a)>v(a-e),
$$

for every $d \in B_{T}(a)$ and $e \notin B_{T}(a)$, that is, $v_{\xi}(y-a)$ is not an element of $v R$, and it induces the cut $(v R \backslash T, T)$ in $v R$. Conversely, if $v_{\xi}(y-a) \in v R(y) \backslash v R$ induces the cut $(v R \backslash T, T)$ in $v R$, then the cut induced by $y-a$ is a ball cut induced by the ball $B_{T}(a)$. 
Suppose that $F$ is any dense subfield of $R$. Consider the following family $\mathcal{F}$ of functions:

$$
\mathcal{F}=\left\{a+b y, \frac{y-a}{y-b} \mid a, b \in F\right\} .
$$

Theorem 2.5. The family $\{U(f) \mid f \in \mathcal{F}\}$ forms a subbasis for the Harrison topology on $M(R(y))$.

Proof. Take any $g \in R(y)$. We will show that $U(g)$ is the union of finite intersections of sets $U(f)$ for suitable $f \in \mathcal{F}$. For this, it is enough to show that, for each $\xi \in U(g)$, there is a finite intersection $U$ of sets $U(f)$ such that $\xi \in U \subset U(g)$.

Let $P$ be an ordering corresponding to $\xi \in U(g)$. We will consider two cases, depending on whether the cut $(D, E)$ corresponding to $P$ is a ball cut or not.

Case 1. Assume that $(D, E)$ is a non-ball cut, i.e., $P$ is the unique ordering determining $\xi$ and $(D, E)$ is a proper cut. Denote by $V_{\xi}$ the value group of the valuation $v_{\xi}$ which corresponds to $\xi$. According to Remark 2.4, we have that $V_{\xi}=v R$. By Lemma 2.1, and since $D \neq \emptyset$ and $E \neq \emptyset$, there are $d \in D$ and $e \in E$ such that $g$ is $\mathbb{Q}^{+}$-bounded on $(d, e)$. Since $(D, E)$ is a non-ball cut, it cannot be principal, i.e., $D$ does not have a last element and $E$ does not have a first element. So we can choose elements $d$ and $e$ closer and closer to the cut, and by the density of $F$ in $R$ we can assume that $d, e \in F$. Suppose first that the following equalities hold:

$$
v_{\xi}(y-d)=v(e-d)=v_{\xi}(e-y) .
$$

Consider the linear functions

$$
f_{1}(y)=\frac{y-d}{e-d} \quad \text { and } \quad f_{2}(y)=\frac{e-y}{e-d},
$$

and set $U=U\left(f_{1}\right) \cap U\left(f_{2}\right)$. We have that $v_{\xi}\left(f_{1}\right)=v_{\xi}\left(f_{2}\right)=0$ and $f_{1}, f_{2} \in P$, so $\xi \in U$. Take any $\eta \in U$ and a cut $\left(D^{*}, E^{*}\right)$ corresponding to any ordering determined by $\eta$. There are $d^{*} \in D^{*}$ and $e^{*} \in E^{*}$ such that $f_{1}$ and $f_{2}$ are $\mathbb{Q}^{+}$-bounded on $\left(d^{*}, e^{*}\right)$. Therefore, $f_{1}(c)>0$ and $f_{2}(c)>0$ for every $c \in\left(d^{*}, e^{*}\right)$. Since both functions $f_{1}$ and $f_{2}$ are positive at the same time only on the interval $(d, e)$, we have that $d \leq d^{*}<e^{*} \leq e$. By Corollary $2.2, \eta \in U(g)$. 
Now suppose that (2.1) does not hold. We shall consider the case of $v(e-d)<v_{\xi}(y-d)$. The case of $v(e-d)<v_{\xi}(e-y)$ is symmetrical. By the density of $F$ in $R$ and the equality $V_{\xi}=v R$, there is a positive $b \in F$ such that $v(b)=v_{\xi}(y-d)$. Set $f(y)=(y-d) / b$ and $U=U(f)$. Then, $v_{\xi}(f)=0$ and $f \in P$, thus $\xi \in U$. Take $\eta \in U$, and let $\left(D^{*}, E^{*}\right)$ be a cut corresponding to any ordering determined by $\eta$. Then there are $d^{*} \in D^{*}$ and $e^{*} \in E^{*}$ such that $f$ is $\mathbb{Q}^{+}$-bounded on $\left(d^{*}, e^{*}\right)$. This yields that $d \leq d^{*}$. Since $v(e-d)<v_{\xi}(y-d)=v(b)$, we have that $v(e-d) / b<0$ which shows that $e$ cannot lie in $\left(d^{*}, e^{*}\right)$ and therefore, $e^{*} \leq e$. Hence, again by Corollary $2.2, \eta \in U(g)$.

Case 2. Assume that $(D, E)$ is a ball cut induced by $B=B_{T}(a)$. Let $\left(D^{\prime}, E^{\prime}\right)$ be the other cut induced by this ball. We can assume that $(D, E)=B^{-}$and $\left(D^{\prime}, E^{\prime}\right)=B^{+}$. First we consider the case of $B \neq R$. By Lemma 2.1, there exist $d \in D, e \in E, d^{\prime} \in D^{\prime}$ and $e^{\prime} \in E^{\prime}$ such that $g$ is $\mathbb{Q}^{+}$-bounded on $(d, e) \cup\left(d^{\prime}, e^{\prime}\right)$. Then, by continuity, $g$ is $\mathbb{Q}^{+}$-bounded on $[d, e] \cup\left[d^{\prime}, e^{\prime}\right]$ as well. Since $B=E \cap D^{\prime}$ is a ball, $D$ does not have a last element and $E^{\prime}$ does not have a first element. Therefore, by the density of $F$ in $R$, we can assume that $d$ and $e^{\prime}$ belong to $F$.

First, assume that $[d, e] \cap\left[d^{\prime}, e^{\prime}\right] \neq \emptyset$, i.e., $g$ is $\mathbb{Q}^{+}$-bounded on $\left[d, e^{\prime}\right]$. Using the density of $F$ in $R$, we choose positive $b_{1}, b_{2} \in F$ such that $v\left(b_{1}\right)=v(a-d)$ and $v\left(b_{2}\right)=v\left(e^{\prime}-a\right)$. We consider the linear functions

$$
f_{1}(y)=\frac{y-d}{b_{1}} \quad \text { and } \quad f_{2}(y)=\frac{e^{\prime}-y}{b_{2}} .
$$

We set $U=U\left(f_{1}\right) \cap U\left(f_{2}\right)$ and take $d_{1}=(d+a) / 2$. Since $d \notin B_{T}(a)$, we have that $v\left(d_{1}-a\right)=v(d-a) / 2=v(d-a)<T$; thus, $d_{1} \notin B_{T}(a)$. Therefore, $d_{1} \in D$. Moreover, $f_{1}\left(d_{1}\right)=(a-d) /\left(2 b_{1}\right)>0$ and $v\left(f_{1}\left(d_{1}\right)\right)=0$, so $\xi\left(f_{1}\left(d_{1}\right)\right) \in \mathbb{R}^{+}$. Similarly, $\xi\left(f_{1}(a)\right) \in \mathbb{R}^{+}$, and since $f_{1}$ is a linear function, we have that $f_{1}\left(d_{1}\right) \leq f_{1}(c) \leq f_{1}(a)$ for every $c \in[(d+a) / 2, a]$. Therefore, $f_{1}$ is $\mathbb{Q}^{+}$-bounded on $((d+a) / 2, a)$. By Lemma 2.1 , this implies that $\xi \in U\left(f_{1}\right)$. In a similar way, one shows that $f_{2}$ is $\mathbb{Q}^{+}$-bounded on $\left(a,\left(a+e^{\prime}\right) / 2\right)$, and therefore, $\xi \in U\left(f_{2}\right)$. We have proved that $\xi \in U$.

Take any $\eta \in U$. Let $\left(D^{*}, E^{*}\right)$ be a cut corresponding to any ordering determined by $\eta$. Then there are $d^{*} \in D^{*}$ and $e^{*} \in E^{*}$ such that $f_{1}$ and $f_{2}$ are $\mathbb{Q}^{+}$-bounded on $\left(d^{*}, e^{*}\right)$. Therefore, $f_{1}(c)>0$ and $f_{2}(c)>0$ 
for every $c \in\left(d^{*}, e^{*}\right)$. This implies that $d \leq d^{*}<e^{*} \leq e^{\prime}$ and, by Corollary 2.2, $\eta \in U(g)$.

Now assume that $[d, e] \cap\left[d^{\prime}, e^{\prime}\right]=\emptyset$. Then $B$ has more than one element. In this case, we can choose the elements $d<e<d^{\prime}<e^{\prime}$ to be in $F$. Consider the two linear functions

$$
f_{1}(y)=\frac{y-d}{e-d} \quad \text { and } \quad f_{2}(y)=\frac{e^{\prime}-y}{e^{\prime}-d^{\prime}}
$$

and the function

$$
f_{3}=\frac{y-e}{y-d^{\prime}}=1+\frac{d^{\prime}-e}{y-d^{\prime}} .
$$

We set $U=U\left(f_{1}\right) \cap U\left(f_{2}\right) \cap U\left(f_{3}\right)$. Since $e, d^{\prime} \in B$, we have that $B=B_{T}(a)=B_{T}(e)=B_{T}\left(d^{\prime}\right)$. Therefore, the same arguments as in the previous case yield that $f_{1}$ is $\mathbb{Q}^{+}$-bounded on $((d+e) / 2, e)$ and $f_{2}$ is $\mathbb{Q}^{+}$-bounded on $\left(d^{\prime},\left(d^{\prime}+e^{\prime}\right) / 2\right)$, whence $\xi \in U\left(f_{1}\right) \cap U\left(f_{2}\right)$. Since $B=B_{T}\left(d^{\prime}\right)$ and $e \in B$, by Remark 2.4, we have that $v\left(e-d^{\prime}\right)>$ $v_{\xi}\left(y-d^{\prime}\right)$. Therefore, we obtain that

$$
v_{\xi}\left(f_{3}\right)=\min \left\{v(1), v_{\xi}\left(\frac{d^{\prime}-e}{y-d^{\prime}}\right)\right\}=0 .
$$

Moreover, $f_{3} \in P$, thus $\xi \in U\left(f_{3}\right)$ and finally $\xi \in U$.

Take $\eta \in U$, and let $\left(D^{*}, E^{*}\right)$ be a cut corresponding to any ordering determined by $\eta$. Then there are $d^{*} \in D^{*}$ and $e^{*} \in E^{*}$ such that $f_{1}$, $f_{2}$ and $f_{3}$ are $\mathbb{Q}^{+}$-bounded on $\left(d^{*}, e^{*}\right)$. Therefore, $f_{1}(c)>0, f_{2}(c)>0$ and $f_{3}(c)>0$ for every $c \in\left(d^{*}, e^{*}\right)$. Since all three functions $f_{1}, f_{2}$ and $f_{3}$ are positive at the same time only on the set $(d, e) \cup\left(d^{\prime}, e^{\prime}\right)$, we find that either $d \leq d^{*}<e^{*} \leq e$ or $d^{\prime} \leq d^{*}<e^{*} \leq e^{\prime}$. By Corollary 2.2, $\eta \in U(g)$.

Now we consider the case of $B=R$, so $D=\emptyset=E^{\prime}$. By Lemma 2.1, there exist $e \in E$ and $d^{\prime} \in D^{\prime}$ such that $g$ is $\mathbb{Q}^{+}$-bounded on $(-\infty, e) \cup\left(d^{\prime}, \infty\right)$. If $(-\infty, e) \cap\left(d^{\prime}, \infty\right) \neq \emptyset$, then we can take $U=U(1)$ since $U=U(g)=U(1)$, and we are done. So let us assume now that $(-\infty, e) \cap\left(d^{\prime}, \infty\right)=\emptyset$. 
Consider the function

$$
f=\frac{y-e}{y-d^{\prime}}=1+\frac{d^{\prime}-e}{y-d^{\prime}}
$$

and set $U=U(f)$. Since $\xi(f)=1$, we have that $\xi \in U$.

Take $\eta \in U$, and let $\left(D^{*}, E^{*}\right)$ be a cut corresponding to any ordering determined by $\eta$. Then there are $d^{*} \in D^{*}$ and $e^{*} \in E^{*}$ such that $f$ is $\mathbb{Q}^{+}$-bounded on $\left(d^{*}, e^{*}\right)$. Therefore, $f(c)>0$ for every $c \in\left(d^{*}, e^{*}\right)$. Since $f$ is positive only on the set $(-\infty, e) \cup\left(d^{\prime}, \infty\right)$, we find that either $d^{*}<e^{*} \leq e$ or $d^{\prime} \leq d^{*}<e^{*}$. By Corollary 2.2, $\eta \in U(g)$.

Remark 2.6. The family $\{U(f) \mid f$ linear polynomial in $F(y)\}$ does not form a subbasis for the Harrison topology on $M(R(y))$. Indeed, take any non-singleton ball $B$ in $R$ and any two elements $e<d^{\prime}$ in $B$. Consider the function $f_{3}$ as in (2.2). We have already shown that the place $\xi$ induced by the ball-cuts generated by $B$ belongs to $U\left(f_{3}\right)$. Suppose there was a finite intersection $U=U\left(g_{1}\right) \cap \cdots \cap U\left(g_{k}\right)$ with linear polynomials $g_{1}, \ldots, g_{k}$ such that $\xi \in U \subseteq U\left(f_{3}\right)$. By Corollary 2.3, $\xi \in U\left(g_{i}\right)$ implies that $g_{i}$ is $\mathbb{Q}^{+}$-bounded on $B$ and hence on $\left\{d^{\prime}\right\}$, for $1 \leq i \leq k$. Now take $\eta$ to be the principal place induced by the ball-cuts generated by the principal ball $\left\{d^{\prime}\right\}$. Again, by Corollary 2.3, $\eta \in U\left(g_{i}\right)$ for $1 \leq i \leq k$, whence $\eta \in U\left(f_{3}\right)$. But $\eta\left(f_{3}\right)=\infty$, a contradiction.

As a corollary to Theorem 2.5 , it follows that the family $\mathcal{F}$ weakly separates points in $M(R(y))$, i.e., if $\xi, \eta \in M(R(y))$ with $\xi \neq \eta$, then there is an $f \in \mathcal{F}$ such that $\xi(f) \neq \eta(f)$. But we will prove an even stronger result. For every $\gamma \in v R=v F$, we choose an element $c_{\gamma} \in F$ such that $v c_{\gamma}=\gamma$. For every $\gamma \in v R$, write $\mathcal{O}_{\gamma}=\{a \in R \mid v a \geq \gamma\}$ and $\mathcal{M}_{\gamma}=\{a \in R \mid v a>\gamma\}$. Then consider a set $\mathcal{S}_{\gamma}^{0}$ of representatives for the cosets in the quotient $R / \mathcal{O}_{\gamma}$ of additive groups, and $\mathcal{S}_{\gamma}$ to be a set of representatives for the cosets in the quotient $R / \mathcal{M}_{\gamma}$. By the density of $F$ in $R$, the sets $\mathcal{S}_{\gamma}^{0}$ and $\mathcal{S}_{\gamma}$ can be chosen as subsets of $F$. We will consider the following subfamilies of $\mathcal{F}$ :

$$
\begin{aligned}
& \mathcal{F}_{0}:=\left\{\frac{y-a}{c_{\gamma}} \mid a \in \mathcal{S}_{\gamma}^{o}, \gamma \in v R\right\} \\
& \mathcal{F}_{1}:=\left\{\frac{y-a}{r c_{\gamma}} \mid a \in \mathcal{S}_{\gamma}, \gamma \in v R\right\} .
\end{aligned}
$$


Proposition 2.7. The family $\mathcal{F}_{0}$ weakly separates points in $M(R(y))$. It is minimal with this property.

Proof. Take $\xi_{1}, \xi_{2} \in M(R(y))$ with $\xi_{1} \neq \xi_{2}$, and take $C_{i}=\left(D_{i}, E_{i}\right)$ to be a cut corresponding to any ordering determined by $\xi_{i}$ for $=1,2$. We can assume that $D_{1} \subset D_{2}$. Since $\xi_{1} \neq \xi_{2}$, we know that $E_{1} \cap D_{2}$ is not a ball. Hence, there are $a_{1}, a_{2} \in E_{1} \cap D_{2}$ and $a_{3} \notin E_{1} \cap D_{2}$ such that

$$
\gamma:=v\left(a_{2}-a_{1}\right) \leq v\left(a_{3}-a_{1}\right) .
$$

Take $a \in \mathcal{S}_{\gamma}^{0}$ to be a representative of $a_{1}+\mathcal{O}_{\gamma}$, and consider the function

$$
f(y)=\frac{y-a}{c_{\gamma}} \in R(y) .
$$

Note that $v\left(a-a_{i}\right) \geq \gamma$ for all $i$. This implies that $v f\left(a_{i}\right) \geq 0$, whence $\xi_{0}\left(f\left(a_{i}\right)\right) \in \mathbb{R}$ for all $i$. One of the cuts $C_{i}$ lies between $a_{1}$ and $a_{3}$; since $f$ is a linear function, this yields that, for the corresponding $i, \xi_{i}(f)$ lies between the values $\xi_{0}\left(f\left(a_{1}\right)\right)$ and $\xi_{0}\left(f\left(a_{3}\right)\right)$ and is therefore itself a real number. If only one of the two values $\xi_{1}(f)$ and $\xi_{2}(f)$ is finite, then we are done. So let us assume that both are finite.

We have that

$$
\begin{aligned}
v\left(f\left(a_{1}\right)-f\left(a_{2}\right)\right) & =v\left(\frac{a_{1}-a}{c_{\gamma}}-\frac{a_{2}-a}{c_{\gamma}}\right) \\
& =v\left(\frac{a_{1}-a_{2}}{c_{\gamma}}\right)=0,
\end{aligned}
$$

so that

$$
\xi_{0}\left(f\left(a_{1}\right)\right) \neq \xi_{0}\left(f\left(a_{2}\right)\right) .
$$

Using this together with the fact that $C_{1}$ lies on the left and $C_{2}$ lies on the right of $a_{1}$ and $a_{2}$, we obtain that

$$
\begin{aligned}
\xi_{1}(f) & \leq \min \left\{\xi_{0}\left(f\left(a_{1}\right)\right), \xi_{0}\left(f\left(a_{2}\right)\right)\right\} \\
& <\max \left\{\xi_{0}\left(f\left(a_{1}\right)\right), \xi_{0}\left(f\left(a_{2}\right)\right)\right\} \\
& \leq \xi_{2}(f),
\end{aligned}
$$

which proves our first assertion.

It remains to prove that the family $\mathcal{F}_{0}$ is minimal with the property of weakly separating points. To show this, it is enough to find, for 
every $f \in \mathcal{F}_{0}$, two places $\xi$ and $\eta \in M(R(y))$ such that $\xi(f) \neq \eta(f)$ and $\xi(g)=\eta(g)$ whenever $f \neq g \in \mathcal{F}_{0}$.

Given

$$
f(y)=\frac{y-a}{c_{\gamma}} \in \mathcal{F}_{0},
$$

we take two balls $B_{T}(a)$ and $B_{T}(b)$, where $b \in R, T=\{\delta \in v R: \delta>\gamma\}$ and $v(a-b)=\gamma$. Then

$$
\xi_{0}\left(\frac{b-a}{c_{\gamma}}\right) \in \mathbb{R} \backslash\{0\} .
$$

Take $\xi$ to be the place corresponding to the cuts $B_{T}(a)^{+}$and $B_{T}(a)^{-}$ and $\eta$ the place corresponding to the cuts $B_{T}(b)^{+}$and $B_{T}(b)^{-}$. Then we have:

$$
\gamma<v_{\xi}(y-a)<\delta \text { and } \gamma<v_{\eta}(y-b)<\delta
$$

for every $\delta>\gamma$, which implies that

$$
\xi\left(\frac{y-a}{c_{\gamma}}\right)=0 \quad \text { and } \quad \eta\left(\frac{y-b}{c_{\gamma}}\right)=0 .
$$

Therefore,

$$
\eta(f)=\eta\left(\frac{y-a}{c_{\gamma}}\right)=\eta\left(\frac{y-b}{c_{\gamma}}+\frac{b-a}{c_{\gamma}}\right)=\xi_{0}\left(\frac{b-a}{c_{\gamma}}\right) \neq 0=\xi(f) .
$$

Now take

$$
g(y)=\frac{y-a^{\prime}}{c_{\gamma^{\prime}}} \in \mathcal{F}_{0}, \quad f \neq g .
$$

We will consider four cases to show that $\xi(g)=\eta(g)$.

Case 1. Assume first that $\gamma=\gamma^{\prime}$. Then $a \neq a^{\prime}$ and, by our choice of $a$, we obtain that $v\left(a-a^{\prime}\right)<\gamma$. It follows that $v_{\xi}\left(y-a^{\prime}\right)=\min \left\{v_{\xi}(y-\right.$ $\left.a), v\left(a-a^{\prime}\right)\right\}=v\left(a-a^{\prime}\right)<\gamma^{\prime}$, and thus, $\xi(g)=\infty$. In a similar way, we obtain that $v_{\eta}\left(y-a^{\prime}\right)=\min \left\{v_{\eta}(y-b), v(b-a), v\left(a-a^{\prime}\right)\right\}=v\left(a-a^{\prime}\right)<$ $\gamma^{\prime}$, so $\xi(g)=\infty$.

Case 2. Assume that $\gamma \neq \gamma^{\prime}$ and $v\left(a-a^{\prime}\right)>\gamma$. Then, we have that $v_{\xi}\left(y-a^{\prime}\right)=\min \left\{v_{\xi}(y-a), v\left(a-a^{\prime}\right)\right\}=v_{\xi}(y-a)$ and $v_{\eta}\left(y-a^{\prime}\right)=\min \left\{v_{\eta}(y-b), v(b-a), v\left(a-a^{\prime}\right)\right\}=\gamma$. By the inequalities (2.3), we obtain that $\xi(g)=\infty=\eta(g)$ if $\gamma^{\prime}>\gamma$ and $\xi(g)=0=\eta(g)$ if $\gamma^{\prime}<\gamma$. 
Case 3. Assume that $\gamma \neq \gamma^{\prime}$ and $v\left(a-a^{\prime}\right)=\gamma$. Then $v_{\xi}\left(y-a^{\prime}\right)=$ $v\left(a-a^{\prime}\right)=\gamma$. Moreover, $v\left(b-a^{\prime}\right) \geq \min \left\{v(b-a), v\left(a-a^{\prime}\right)\right\}=\gamma$. If $v\left(b-a^{\prime}\right)>\gamma$, then $v_{\eta}\left(y-a^{\prime}\right)=\min \left\{v_{\eta}(y-b), v\left(b-a^{\prime}\right)\right\}=v_{\eta}(y-b)$. If $v\left(b-a^{\prime}\right)=\gamma$, then $v_{\eta}\left(y-a^{\prime}\right)=\min \left\{v_{\eta}(y-b), v\left(b-a^{\prime}\right)\right\}=\gamma$. In both subcases, again using (2.3) and distinguishing the cases of $\gamma^{\prime}>\gamma$ and $\gamma^{\prime}<\gamma$ as in Case 2, one shows that $\xi(g)=\eta(g)$.

Case 4. Assume that $\gamma \neq \gamma^{\prime}$ and $v\left(a-a^{\prime}\right)<\gamma$. Then $v_{\xi}\left(y-a^{\prime}\right)=$ $v\left(a-a^{\prime}\right)$ and $v_{\eta}\left(y-a^{\prime}\right)=v\left(a-a^{\prime}\right)$. If $v\left(a-a^{\prime}\right) \neq \gamma^{\prime}$, then $\xi(g)=\infty=\eta(g)$ if $\gamma^{\prime}>\gamma$ and $\xi(g)=0=\eta(g)$ if $\gamma^{\prime}<\gamma$. So, assume now that $v\left(a-a^{\prime}\right)=\gamma^{\prime}$. Then, also $v\left(b-a^{\prime}\right)=\gamma^{\prime}$. Since $\gamma^{\prime}=v\left(a-a^{\prime}\right)<\gamma=v(b-a)$, we have that $\xi_{0}(b-a) / c_{\gamma^{\prime}}=0$. By the inequalities (2.3), we get that

$$
\xi\left(\frac{y-a}{c_{\gamma^{\prime}}}\right)=0=\eta\left(\frac{y-b}{c_{\gamma^{\prime}}}\right) .
$$

Thus,

$$
\begin{aligned}
\eta(g) & =\eta\left(\frac{y-a^{\prime}}{c_{\gamma^{\prime}}}\right)=\eta\left(\frac{y-b}{c_{\gamma^{\prime}}}+\frac{b-a^{\prime}}{c_{\gamma^{\prime}}}\right)=\xi_{0}\left(\frac{b-a^{\prime}}{c_{\gamma^{\prime}}}\right) \\
& =\xi_{0}\left(\frac{b-a}{c_{\gamma^{\prime}}}+\frac{a-a^{\prime}}{c_{\gamma^{\prime}}}\right)=\xi_{0}\left(\frac{a-a^{\prime}}{c_{\gamma^{\prime}}}\right)=\xi\left(\frac{y-a}{c_{\gamma^{\prime}}}+\frac{a-a^{\prime}}{c_{\gamma^{\prime}}}\right) \\
& =\xi\left(\frac{y-a^{\prime}}{c_{\gamma^{\prime}}}\right)=\xi(g) .
\end{aligned}
$$

This completes the proof of our proposition.

Proposition 2.8. The family $\mathcal{F}_{1}$ separates points in $M(R(y))$ in the topological sense, that is, if $\xi_{1}, \xi_{2} \in M(R(y))$ with $\xi_{1} \neq \xi_{2}$, then there is an $f \in \mathcal{F}$ such that $\xi_{1} \notin U(f) \ni \xi_{2}$ or $\xi_{2} \notin U(f) \ni \xi_{1}$.

Proof. In the proof of Proposition 2.7, we constructed the function $f(y)=(y-a) / c_{\gamma}$ such that only one of the two values $\xi_{1}(f)$ and $\xi_{2}(f)$ is finite or $\xi_{1}(f)<\xi_{2}(f)$ if both are finite.

Take any rational number $q$, and denote by $\widetilde{a} \in \mathcal{S}_{\gamma}$ the representative of $a+q c_{\gamma}$ modulo $\mathcal{M}_{\gamma}$. We set

$$
\widetilde{f}(y):=\frac{y-\widetilde{a}}{c_{\gamma}} .
$$


Since $v\left(\widetilde{a}-\left(a+q c_{\gamma}\right)>\gamma\right.$, for every place $\xi \in M(R(y))$, we have that

$$
\begin{aligned}
\xi\left(\frac{y-\widetilde{a}}{c_{\gamma}}\right) & =\xi\left(\frac{y-\left(a+q c_{\gamma}\right)}{c_{\gamma}}\right) \\
& =\xi\left(\frac{y-a}{c_{\gamma}}\right)+\xi\left(\frac{-q c_{\gamma}}{c_{\gamma}}\right) \\
& =\xi(f)-q .
\end{aligned}
$$

If $\xi_{1}(f)$ and $\xi_{2}(f)$ are both finite, then we choose $q$ such that $\xi_{1}(f)<$ $q<\xi_{2}(f)$ and obtain that

$$
\xi_{1}(\widetilde{f})<0<\xi_{2}(\widetilde{f}) .
$$

If $\xi(f)$ is infinite, then by $(2.4), \xi(\widetilde{f})$ is also infinite. Hence, in the case of $\xi_{1}(f)$ finite and $\xi_{2}(f)$ infinite, we choose $q<\xi_{1}(f)$ to obtain that $\xi_{1}(\widetilde{f})>0$ and $\xi_{2}(\widetilde{f})$ is infinite. For the remaining case, we interchange the role of $\xi_{1}$ and $\xi_{2}$.

3. The metrizable case. We assume that $M(R(y))$ is metrizable, which is equivalent to the existence of a countable real closed field $F$ that lies dense in $R$ (see [8, Theorem 4.7]).

Since $F(y)$ is a countable field and since a subbasis for the space $M(F(y))$ is defined by the elements of the field $F(y)$, the space $M(F(y))$ is second-countable, and, by Urysohn's metrization theorem, is metrizable. On the other hand, one can see $M(F(y))$ as a closed subset of the space $\overline{\mathbb{R}}^{F(y)}$, where $\overline{\mathbb{R}}=\mathbb{R} \cup\{\infty\}$ is the circle (see [1]). Therefore, the topology on $M(F(y))$ is induced by the restriction of the canonical metric of the product $\overline{\mathbb{R}}^{F(y)}$. According to [3, Chapter IX, Theorem 7.2], this metric can be defined in the following way. First, choose any bijection $\sigma: F(y) \rightarrow \mathbb{N} \cup\{0\}$. Then, the metric $\rho: M(F(y)) \times M(F(y)) \rightarrow[0, \infty)$ is given by

$$
\rho(\xi, \eta)=\sup _{f \in F(y)}\left\{2^{-\sigma(f)} d_{0}(\xi(f), \eta(f))\right\},
$$

where $d_{0}$ is any fixed metric on the circle $\overline{\mathbb{R}}$.

Let $\mathcal{F}$ be the family of functions defined in Section 2. We will show that, in the above definition of the metric, one can restrict the functions $f$ to the family $\mathcal{F}$. The mapping $d: M(F(y)) \times M(F(y)) \rightarrow[0, \infty)$, 
given by:

$$
d(\xi, \eta)=\sup _{f \in \mathcal{F}}\left\{2^{-\sigma(f)} d_{0}(\xi(f), \eta(f))\right\},
$$

is symmetric, and the triangle inequality holds since it holds for $d_{0}$. By Proposition 2.7, we have $d(\xi, \eta)=0$ if and only if $\xi=\eta$, so $d$ is a metric on $M(F(y))$.

Lemma 3.1. For every $f \in \mathcal{F}$ and every $\xi \in U(f)$. There is a $\delta>0$ such that, if $d(\xi, \eta)<\delta$, then $\eta \in U(f)$, i.e., the metric ball $B_{d}(\xi, \delta)$ is contained in $U(f)$.

Proof. Take $f \in \mathcal{F}$ and $\xi \in U(f)$. We have that $\xi(f) \in(0, \infty)$. Set

$$
\delta=2^{-\sigma(f)} \min \left\{d_{0}(\xi(f), 0), d_{0}(\xi(f), \infty)\right\} .
$$

If $d(\xi, \eta)<\delta$, then also $2^{-\sigma(f)} d_{0}(\xi(f), \eta(f))<\delta$. Thus,

$$
d_{0}(\xi(f), \eta(f))<\min \left\{d_{0}(\xi(f), 0), d_{0}(\xi(f), \infty)\right\}
$$

and therefore, $\eta(f) \in(0, \infty)$, so $\eta \in U(f)$.

Proposition 3.2. The Harrison topology of the space $M(F(y))$ is equal to the topology introduced by the metric d defined above.

Proof. Lemma 3.1 shows that every subbasic set $U(f)$, for $f \in \mathcal{F}$, is open in the topology induced by the metric $d$. Hence, this topology is finer than or equal to the Harrison topology. For the reverse, note that, since $\mathcal{F} \subset F(y)$, the definitions of the metrics $d$ and $\rho$ give us that $B_{\rho}(\xi, \epsilon) \subset B_{d}(\xi, \epsilon)$. Take $\eta \in B_{d}(\xi, \epsilon)$. We have that, for some $\delta<\epsilon$, $B_{d}(\eta, \delta) \subset B_{d}(\xi, \epsilon)$, whence $B_{\rho}(\eta, \delta) \subset B_{d}(\eta, \delta) \subset B_{d}(\xi, \epsilon)$. Therefore, $B_{d}(\xi, \epsilon)$ is open in the topology induced by $\rho$, that is, the Harrison topology is finer than or equal to the topology induced by $d$.

By [8, Theorem 3.2], the restriction mapping

$$
\text { res }: M(R(y)) \longrightarrow M(F(y)), \quad \operatorname{res}(\xi)=\left.\xi\right|_{F(y)}
$$

is a homeomorphism. By this homeomorphism, the metric $d$ induces the metric on $M(R(y))$. We have proved: 
Theorem 3.3. Let $R$ be a real closed field and $F$ be a countable, real closed, dense subfield of $R$. Set

$$
\mathcal{F}=\left\{a+b y, \frac{y-a}{y-b} \mid a, b \in F\right\} .
$$

Take any bijection $\sigma: \mathcal{F} \rightarrow \mathbb{N}$. Then the mapping $d: M(R(y)) \times$ $M(R(y)) \rightarrow[0, \infty)$ given by

$$
d(\xi, \eta)=\sup _{f \in \mathcal{F}}\left\{2^{-\sigma(f)} d_{0}(\xi(f), \eta(f))\right\}
$$

is a metric on $M(R(y))$.

4. The dimension of $M(R(y))$. In the paper [1], the authors evaluated the dimensions of the spaces of $\mathbb{R}$-places of a rational function field $L=K\left(x_{1}, \ldots, x_{n}\right)$ over a totally archimedean field $K$. They proved that the covering dimension (dim) of such spaces is bounded from above by $n$. Moreover, for $n \leqslant 2, \operatorname{dim}(M(L))=n$. The authors also proved that the cohomological dimension of $M\left(K\left(x_{1}, x_{2}\right)\right)$ is equal to 1 , which shows that this space is not dimensionally full-valued. The methods used in the proofs of these results strongly rely on the fact that $K$ is a totally archimedean field and cannot be extended to general case.

In the present paper, we will determine the dimension of the space $M(R(y))$ for any real closed field $R$. We consider the covering dimension dim, the small inductive dimension ind, and the strong inductive dimension Ind. The following result is part of [11, Theorem 5]:

Theorem 4.1. If $Y$ is the continuous image of a compact ordered space, then $\operatorname{dim} Y=\operatorname{ind} Y=\operatorname{Ind} Y$.

Since the space $M(R(y))$ is the continuous image under the mapping (1.1) of the compact space $\mathcal{X}(R(y))$, which is ordered through the homeomorphism with totally ordered space $\mathcal{C}(R)$ of cuts in $R$ given in Proposition 1.1, we obtain the following result:

Corollary 4.2. We have that

$$
\operatorname{dim} M(R(y))=\text { ind } M(R(y))=\operatorname{Ind} M(R(y)) .
$$


The space $M(R(y))$ is connected (see [5]). That gives us a lower bound for the dimension of that space; it is at least 1 . To obtain an upper bound, we use the following theorem (cf., [10, Theorem III.7]):

Theorem 4.3. Let $f$ be a continuous mapping of a space $X$ onto a space $Y$ such that, for each point $\eta$ of $Y$, the boundary of $f^{-1}(\eta)$ contains at most $m+1$ points $(m \geq 1)$. Then $\operatorname{dim} Y \leq \operatorname{dim} X+m$.

We apply the theorem to $\lambda: \mathcal{X}(R(y)) \rightarrow M(R(y))$. For every $\eta \in M(R(y)), \lambda^{-1}(\eta)$ contains at most two points and is closed, so its boundary contains at most two points. On the other hand,

$$
\operatorname{dim} \mathcal{X}(R(y))=\operatorname{Ind} \mathcal{X}(R(y))=0,
$$

since $\mathcal{X}(R(y))$ is totally disconnected. The last theorem now shows that $\operatorname{dim} M(R(y)) \leq 1$. Putting everything together, we obtain the equation

$$
1=\operatorname{dim} M(R(y))=\operatorname{ind} M(R(y))=\operatorname{Ind} M(R(y)),
$$

which proves Theorem 1.3.

5. Homeomorphisms between subspaces of $M(R(y))$. If $L$ is any field, then every automorphism $\sigma$ of $L$ induces the following bijection of $M(L)$ onto itself:

$$
M(L) \ni \xi \longmapsto \xi \circ \sigma \in M(L) .
$$

This bijection is in fact a homeomorphism because

$$
\xi \in U(b) \Longleftrightarrow \xi \circ \sigma \in U\left(\sigma^{-1} b\right) .
$$

Let us have a closer look at the case of $M(R(y))$, with $R$ a real closed field. It is well known that the automorphisms $\sigma$ of $R(y)$ which leave $R$ element-wise fixed are precisely those given by

$$
y \longmapsto \frac{a y+b}{c y+d} \quad \text { with } a d-b c \neq 0 .
$$

We can study the effect of the homeomorphism of $M(R(y))$ induced by such an automorphism by analyzing the corresponding effect on $\mathcal{C}(R)$. 
If $c \neq 0$, then

$$
\frac{a y+b}{c y+d}=\frac{a}{c}+\frac{b-\frac{a d}{c}}{c y+d} .
$$

Hence, the assignment (5.1) can always be achieved by a composition of addition of, and multiplication by, elements from $R$ together with one inversion of a linear polynomial in $y$. The corresponding actions on $\mathcal{C}(R)$ can be described as follows. Fix an ordering on $R(y)$, take $z \in R(y)$ such that $R(y)=R(z)$, and let $C=(D, E)$ be the cut induced by $z$ in $R$.

(1) The automorphism induced by $z \mapsto z+c$ for some $c \in R$. The cut induced by $z+c$ in $R$ is the shifted cut $C+c=(D+c, E+c)$. If $C=(D, E)$ is a ball cut generated by the ball $B_{T}(a)$, then $C+c$ is a ball cut generated by the ball $B_{T}(a+c)$.

(2) The automorphism induced by $z \mapsto c z$ for some $c \in R \backslash\{0\}$. The cut induced by $c z$ in $R$ is the cut $c C=(c D, c E)$ if $c>0$, and $c C=(c E, c D)$ if $c<0$. If $C$ is a ball cut generated by the ball $B_{T}(a)$, then $c C$ is a ball cut generated by the ball $B_{T+v(c)}(c a)$ where $T+v(c)$ is the final segment $\{t+v(c) \mid t \in T\}$.

(3) The automorphism induced by $z \mapsto z^{-1}$. Denote the cut induced by $z^{-1}$ in $R$ by $C^{\prime}$. If $z>0$, then

$$
C^{\prime}=\left(\left\{d \mid d \leq 0 \text { or } d^{-1} \in E\right\},\left\{e>0 \mid e^{-1} \in D\right\}\right) .
$$

If $z<0$, then

$$
C^{\prime}=\left(\left\{d<0 \mid d^{-1} \in E\right\},\left\{e \mid e \geq 0 \text { or } e^{-1} \in D\right\}\right) .
$$

In particular, this shows that, if $C$ is a ball cut generated by the ball $\{0\}$, then $C^{\prime}$ is a ball cut generated by the ball $R$, and vice versa.

Now assume that $C$ is a ball cut generated by a ball $B_{T}(a)$ different from $\{0\}$ and $R$. Let us first discuss the case where $0 \in B_{T}(a)$, so we can take $a=0$. Then, by Remark 2.4, $v(z)$ induces the cut $(S, T)$ in $v R$, and it follows that $v\left(z^{-1}\right)=-v(z)$ induces the cut $(-T,-S)$. Consequently, again by Remark $2.4, C^{\prime}$ is a ball cut generated by the ball $B_{-S}(0)$.

Finally, we deal with the case of $0 \notin B_{T}(a)$. Then, again from Remark 2.4, it follows that $v z \in v R$ and $v(z-a) \notin v R$. This yields 
that $v(z-a)>v z=v a$ and

$$
v\left(\frac{1}{z}-\frac{1}{a}\right)=v\left(\frac{a-z}{a z}\right)=v(z-a)-2 v(a) \notin v R .
$$

The value $v(z-a)-2 v(a)$ induces the cut in $v R$ that has upper cut set $T-2 v(a)$. Consequently, by Remark $2.4, C^{\prime}$ is a ball cut generated by the ball $B_{T-2 v(a)}\left(a^{-1}\right)$.

Note that the inverses of each of these actions are again of the same form. The first two actions are either order preserving or order reversing on $R$, and the last is order reversing on $\{r \in R \mid r<0\}$ and on $\{r \in R \mid r>0\}$. Therefore, they induce homeomorphisms on $\mathcal{C}(R)$. We summarize:

Proposition 5.1. All three actions induce homeomorphisms on $\mathcal{C}(R)$ that are compatible with equivalence.

As we see already from the above discussion, the balls in $R$ are crucial for the analysis of the homeomorphisms between subspaces of $M(R(y))$. So let us make the link precise. For any set $S \subseteq R$, we define $\widehat{S}$ to be the closure of the set $\left\{a^{-}, a^{+} \mid a \in S\right\}$ in $\mathcal{C}(R)$. If $S$ is an interval in $R$, then $\widehat{S}$ is a closed interval in $\mathcal{C}(R)$. If $S=B$ is a ball in $R$, then $\widehat{S}=\left[B^{-}, B^{+}\right]$. If $S$ is a ball complement $B^{c}:=R \backslash B$ for some ball $B$, then $\widehat{S}=\left[R^{-}, B^{-}\right] \cup\left[B^{+}, R^{+}\right]$.

Further, we send $\widehat{S}$ to

$$
\bar{S}:=\lambda \circ \chi(\widehat{S}) \subseteq M(R(y)),
$$

which is the set of all the $\mathbb{R}$-places induced by cuts in $\widehat{S}$. Let us call a subset of $M(R(y))$ a ball (in $M(R(y))$ ) if it is the image $\bar{B}$ or $\overline{B^{c}}$ of a ball or ball complement in $R$. We can view the latter as the balls around the place at infinity.

Now the homeomorphisms between subspaces of $M(R(y))$ can be analyzed by determining which balls in $R$ can be sent onto each other by homeomorphisms of $M(R(y))$. Take $a, b \in R, \gamma \in v R$ and a final segment $T$ of $v R$. Then, by a combination of actions (1) and (2) above, $B_{T}(a)$ can be sent onto $B_{T+\gamma}(b)$ and $B_{T}(a)^{c}$ can be sent onto $B_{T+\gamma}(b)^{c}$, so the corresponding balls in $M(R(y))$ can be sent onto each other by a homeomorphism. Further, action (3) transforms an interval 
$\left[B_{T}(0)^{-}, B_{T}(0)^{+}\right]$in $\mathcal{C}(R)$ into the set $\left[R^{-}, B_{-S}(0)^{-}\right] \cup\left[B_{-S}(0)^{+}, R^{+}\right]$, where $S=v R \backslash T$. The image of this set in $M(R(y))$ is the ball $\overline{B_{-S}(0)^{c}}$ around infinity. In particular, for every $\gamma \in v R$, we have the following sequence of homeomorphisms, which we will interpret later:

$$
\begin{aligned}
\overline{B_{\gamma^{+}}(b)} & \longrightarrow \overline{B_{\gamma^{+}}(0)} \longrightarrow \overline{B_{\gamma^{+}-2 \gamma}(0)} \\
& =\overline{B_{(-\gamma)^{+}}(0)} \longrightarrow \overline{B_{\gamma^{-}}(0)^{c}} \longrightarrow \overline{B_{\gamma^{-}}(a)^{c}} .
\end{aligned}
$$

For any $r \in R$, the set

$$
\left\{\overline{B_{\gamma^{-}}(r)} \mid \gamma \in v R\right\}
$$

forms a cofinal and coinitial chain of subspaces of $M(R(y))$ which are all mutually homeomorphic, and whose intersection only contains the $r$ principal place. The order type of this chain is equal to that of $v R$. The same is true for the chains $\left\{\overline{B_{\gamma^{+}}(r)} \mid \gamma \in v R\right\}$ and $\left\{\overline{B_{T+\gamma}(r)} \mid \gamma \in v R\right\}$.

So far, we have discussed homeomorphisms of $M(R(y))$ that transform it onto itself. The question arises whether there are also homeomorphisms onto proper subspaces, like zooming in on a fractal substructure. How "homogeneous" is the space $M(R(y))$ ? For example, can such chains as in (5.3) be taken so that all spaces in it are homeomorphic to $M(R(y))$ ? Moreover, the following question appears to be of importance when the spaces of $\mathbb{R}$-places of finite extensions of $R(y)$ are studied:

Open problem. If $B$ is a non-singleton ball in $R$, is there a homeomorphism from $\mathcal{C}(R)$ onto $\widehat{B}$ that is compatible with equivalence? More generally, give a criterion for two non-singleton balls $B$ and $B^{\prime}$ in $R$ to admit a homeomorphism from $\widehat{B}$ onto $\widehat{B^{\prime}}$ that is compatible with equivalence.

Such homeomorphisms would induce interesting homeomorphisms of $M(R(y))$ onto proper subspaces $\bar{B}$. Indeed, if $S$ is any subset of $R$ and $\widehat{\psi}: \mathcal{C}(R) \rightarrow \widehat{S}$ a homeomorphism compatible with equivalence, then we obtain an induced homeomorphism $\bar{\psi}$ such that the following diagram commutes: 


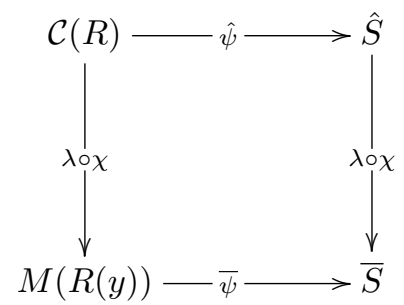

For the conclusion of this section, we wish to give an example of a real closed field $R$ for which there exist homeomorphisms from $M(R(y))$ onto infinitely many distinct subspaces.

Consider the power series field $R=\mathbb{R}\left(\left(t^{\mathbb{Q}}\right)\right)$ with coefficients in $\mathbb{R}$ and exponents in $\mathbb{Q}$. This is a real closed field ([4, Theorem 4.3.7]). Since any two countable dense linear orderings without endpoints are order isomorphic, there exists an order isomorphism $\varphi_{T}$ from $\mathbb{Q}$ onto any non-empty final segment $T$ of $\mathbb{Q}$ which does not have a smallest element. Any such isomorphism induces an isomorphism

$$
\psi_{T}: R=\mathbb{R}\left(\left(t^{\mathbb{Q}}\right)\right) \ni \sum_{q \in \mathbb{Q}} c_{q} t^{q} \longmapsto \sum_{q \in \mathbb{Q}} c_{q} t^{\varphi_{T}(q)} \in B_{T}(0)
$$

from the ordered additive group of $R$ onto its convex subgroup $B=$ $B_{T}(0)$. This isomorphism induces a homeomorphism $\widehat{\psi_{T}}: \mathcal{C}(R) \rightarrow$ $\widehat{B_{T}(0)}$ which is compatible with equivalence. Indeed, if $T^{\prime}$ is a final segment of $\mathbb{Q}$, then $\varphi_{T}\left(T^{\prime}\right)$ is a final segment of $\mathbb{Q}$ contained in $T$; hence, the image of the ball $B_{T^{\prime}}(a)$ under $\psi_{T}$ is the ball $B_{\varphi_{T}\left(T^{\prime}\right)}\left(\psi_{T}(a)\right)$.

If $r$ is any element in $R$, then we can compose the homeomorphism $\widehat{\psi_{T}}$ with the homeomorphism that sends $\widehat{B_{T}(0)}$ to $\widehat{B_{T}(r)}$, in order to obtain a homeomorphism

$$
\overline{\psi_{T, r}}: M(R(y)) \longrightarrow \overline{B_{T}(r)} .
$$

As the non-empty final segments $T$ of $\mathbb{Q}$ without smallest element form a dense linear ordering under inclusion, and since their intersection is empty, we obtain:

Theorem 5.2. Take the field $R=\mathbb{R}\left(\left(t^{\mathbb{Q}}\right)\right)$ and $r \in R$. Then there exists a set of subspaces of $M(R(y))$, all homeomorphic to $M(R(y))$, 
on which inclusion induces the dense linear order of $v R$, and such that the $r$-principal place is the only $\mathbb{R}$-place of $R$ contained in all of them.

Based on this theorem we will now show that the space $M(R(y))$ for $R=\mathbb{R}\left(\left(t^{\mathbb{Q}}\right)\right)$ is even self-homeomorphic, in the sense of [2], which means that every open subset contains a homeomorphic copy of $M(R(y))$. To this end, we prove:

Lemma 5.3. Take an arbitrary real closed field $R$. Then every nonempty open subset of $M(R(y))$ contains $\overline{B_{\gamma^{+}}(r)}$ for some $\gamma \in v R$ and $r \in R$.

Proof. Take a nonempty open subset $U$ of $M(R(y))$. Its preimage $(\lambda \circ \chi)^{-1}(U)$ in $\mathcal{C}(R)$ is nonempty open and thus contains an nonempty open interval $\left(C_{1}, C_{2}\right)$. Writing $C_{1}=\left(D_{1}, E_{1}\right)$ and $C_{2}=\left(D_{2}, E_{2}\right)$, we find that $E_{1} \cap D_{2} \neq \emptyset$ must contain more than one element since, otherwise, we would have $C_{1}=a^{-}$and $C_{2}=a^{+}$and the interval would be empty. Therefore, we can choose distinct elements $a, b \in E_{1} \cap D_{2}$. We set $\gamma=v(a-b)$ and $r=(a+b) / 2$. Then $B_{\gamma^{+}}(r) \subset E_{1} \cap D_{2}$, and thus, $\overline{B_{\gamma^{+}}(r)} \subset U$.

Using (5.4) with $T=\gamma^{+}$, we obtain:

Corollary 5.4. For $R=\mathbb{R}\left(\left(t^{\mathbb{Q}}\right)\right)$, the space $M(R(y))$ is self-homeomorphic.

6. The "densely fractal pearl necklace". Take any non archimedean real closed field $R$. In this section, we describe the fractal structure of $M(R(y))$. We start with the linearly ordered set $\mathcal{C}(R)$. For every element $a \in R$, there are the two principal cuts $a^{-}$and $a^{+}$in $\mathcal{C}(R)$. But these are glued by $\lambda \circ \chi$, so we obtain a canonical embedding of $R$ in $M(R(y))$ whose image is exactly the set of principal places. Also, the cuts $R^{-}$and $R^{+}$are glued by $\lambda \circ \chi$, which closes the linear ordering of the principal places at the ends, making it into a (non-archimedean) circle. If we started with $R=\mathbb{R}$, these are already all possible gluings, and we have obtained the usual circle. If $R \neq \mathbb{R}$, we have to go on.

In the circular structure we have obtained, the principal places are joined by the images of the non-ball cuts, on which $\lambda \circ \chi$ is injective, 
that is, which are not glued with other cuts. If $R$ is a real closed subfield of $\mathbb{R}$, the first step has put all elements of $R$ in the circle, while this second step has added all elements of $\mathbb{R}$, and we are done, again having obtained the usual circle.

From now on, we assume that $R$ is any non-archimedean real closed field. By the first two steps, we have obtained what at first glance appears to be the circular string of our necklace.

Sitting densely between the non-gluing and principal cuts are the ball-cuts. Each gluing of two cuts $B^{-}$and $B^{+}$splits the necklace open and forms from a part of it a smaller "circle"-a ball in our necklace. But as $B=B_{T}(a)$ for a final segment with $T \neq \emptyset, v R$ of $v R$, there are final segments $T^{\prime}, T^{\prime \prime} \neq \emptyset, v R$ such that $T^{\prime} \subsetneq T \subsetneq T^{\prime \prime}$. It follows that $B_{T^{\prime \prime}}(a) \subsetneq B_{T}(a) \subsetneq B_{T^{\prime}}(a)$, and the same happens around every other $b \in R$. This shows that each ball is made up of smaller balls and is itself part of a larger ball.

It should be noted that gluings do not "cross" each other; this is because if two balls have a non-empty intersection, then one of the balls is contained in the other.

Our understanding of a necklace usually is that there is a thread that holds together pearls. So let us look for subspaces of $M(R(y))$ which resemble this pattern. For each $\gamma \in v R$ and $a \in R$, we will call $\overline{B_{\gamma^{-}}(a)}$ a subnecklace of $M(R(y))$. We observe that

$$
B_{\gamma^{-}}(a)=\bigcup_{b \in B_{\gamma^{-}}(a)} B_{\gamma^{+}}(b)
$$

so we will call $\overline{B_{\gamma^{+}}(b)}$ a pearl of $\overline{B_{\gamma^{-}}(a)}$. Then we can take the thread to be $\bar{S}$ for any set $S \subset B_{\gamma^{-}}(a)$ of representatives of the subsets $B_{\gamma^{+}}(b)$.

Let us have a closer look at the subnecklace $\overline{B_{\gamma^{-}}(a)}$. It contains the place that is induced by the equivalent ball cuts $B_{\gamma^{-}}(a)^{-}$and $B_{\gamma^{-}}(a)^{+}$. This place is not contained in any of the pearls $\overline{B_{\gamma^{+}}(b)}$. It can be seen as the point through which the subnecklace is connected to the rest of $M(R(y))$. This, however, can itself be viewed as a pearl of the subnecklace $\overline{B_{\gamma^{-}}(a)}$. This is because, by $(5.2), \overline{B_{\gamma^{-}}(a)^{c}}$ is homeomorphic to every pearl $\overline{B_{\gamma^{+}}(b)}$. 
We see that if we apply a homeomorphism sending $\overline{B_{\gamma^{-}}(a)}$ to $\overline{B_{\delta^{-}}\left(a^{\prime}\right)}$, we zoom from one subnecklace to another. That is why we talk of a "fractal pearl necklace." However, there is a difference to the well known fractal structures. Since $R$ is real closed, its value group $v R$ is divisible and hence dense. Therefore, the final segments of $v R$, ordered by inclusion, also form a dense linearly ordered set. Every final segment corresponds to a "level" of subnecklaces, a level of the fractal structure. So for each level, there is no immediate predecessor or successor; when we pass from one subnecklace to a bigger or smaller one we automatically jump through infinitely many intermediate levels. This is why we call $M(R(y))$ "densely fractal."

We also observe that, by our definition, $M(R(y))$ is actually not itself a necklace, as it is not of the form $\overline{B_{\gamma^{-}}(a)}$. It is rather the union of an infinite ascending chain (5.3) of subnecklaces. The situation is the same around every principal place, represented by an element $r \in R$. Switching from one element $r$ to another can be considered as turning the necklace, or more precisely, turning subnecklaces and pearls at infinitely many levels. This is a fractal rotational symmetry along the string(s) of principal and non-glued places.

It is not necessarily true that each level is perfectly similar to every other level. For instance, the balls in $R$ can have different cofinalities. The more homogeneous the field $R$ is, the more homeomorphisms we will obtain between balls in $M(R(y))$. If the field $R$ is sufficiently homogeneous, as is the case for the field $\mathbb{R}\left(\left(t^{\mathbb{Q}}\right)\right)$ which we discussed in the previous section, then around every principal place there will even be a coinitial and cofinal chain of subspaces at all levels that are all homeomorphic to $M(R(y))$ (cf., Theorem 5.2).

\section{REFERENCES}

1. T. Banakh, Ya. Kholyavka, K. Kuhlmann, M. Machura and O. Potyatynyk, The dimension of the space of $\mathbb{R}$-places of certain rational function fields, Cent. Europ. J. Math. 12 (2014), 1239-1248.

2. W.J. Charatonik and A. Dilks, On self-homeomorphic spaces, Topol. Appl. 55 (1994), 215-238.

3. J. Dugundji, Topology, Allyn and Bacon, Boston, 1966.

4. A.J. Engler and A. Prestel, Valued fields, Springer Monogr. Math., SpringerVerlag, Berlin, 2005.

5. J. Harman, Chains of higher level orderings, Contemp. Math. 8 (1982), 141174. 
6. F.-V. Kuhlmann and K. Kuhlmann, Embedding theorems for spaces of $\mathbb{R}$ places of rational function fields and their products, Fund. Math. 218 (2012), 121149.

7. F.-V. Kuhlmann, S. Kuhlmann, M. Marshall and M. Zekavat, Embedding ordered fields in formal power series fields, J. Pure Appl. Algebra 169 (2002), 7190 .

8. F.-V. Kuhlmann, M. Machura and K. Osiak, Spaces of $\mathbb{R}$-places of function fields over real closed fields, Comm. Algebra 39 (2011), 3166-3177.

9. T.Y. Lam, Orderings, valuations and quadratic forms, CBMS Reg. Conf. 52, published for the Conf. Board Math. Sciences, Washington, 1983.

10. J. Nagata, Modern dimension theory, Bibliotheca Math. 6, Interscience Publishers, John Wiley \& Sons, Inc., New York, 1965.

11. J. Nikiel, H.M. Tuncali and E.D. Tymchatyn, On the rim-structure of continuous images of ordered compacta, Pac. J. Math. 149 (1991), 145-155.

Institute of Mathematics, Silesian University, Bankowa 14, 40-007 Katowice, Poland

Email address: kmk@math.us.edu.pl 